\section{The role of proteomics in investigating psychiatric}

\section{disorders}

\author{
K. PENNINGTON, D. COTTER and M. J. DUNN
}

\begin{abstract}
Summary Proteomics is the study of protein expression and function on a genome-wide scale and its application in psychiatric research is relatively new. As protein expression is the mediator of genetic vulnerabilities, it is critical that this area is explored further to increase our understanding of psychiatric disease and reveal potential new treatments.
\end{abstract}

\section{Declaration of interest None. \\ Funding detailed in Acknowledgements.}

The translation of genetic information to proteins forms the basis for most metabolic ecules are required, genes are transcribed first to the corresponding messenger ribonucleic acids (mRNAs) and then translated to their protein counterparts. Additional protein diversity is introduced by subsequent processes of post-translational modification (e.g. phosphorylation, glycosylation), resulting in the biological phenotype. This process of transcription, translation and post-translational modification is variable, depending on genetic predispositions and environmental interactions. Thus, the previous notion that one gene results in one protein no longer holds true. Indeed it has been estimated that, if the human genome contains 30000 expressed genes, there may be the different proteins. Bearing this in mind, the prospect of identifying what is now termed the 'proteome' (the protein complement of a genome (Wasinger et al, 1995)) is daunting. Fortunately, the birth of 'proteomics', an umbrella term encompassing the many tools available to investigate proteins expressed within cells, fluids, tissues or manageable. These proteomic methods have the power to display and quantify the functional expression of genes, enabling processes in the body. When these molpotential to express as many as one million organisms, has made this task more the measurement of disease-associated or phenotypic changes in proteins.

Proteomic studies thus have the potential to provide important insights into our understanding of psychiatric, neurodegenerative and neurological diseases. For example, by identifying alterations in protein expression in post-mortem brain they can provide new insights into the cellular mechanism underlying diseases such as schizophrenia. Likewise, by characterising disease-associated protein profiles in sera they may be able to identify and quantify proteins that serve as diagnostic or prognostic markers. Furthermore, proteomic investigation of the effects of pharmaceutical agents in vitro may help us to identify new therapeutic pathways. In this context, it is of interest to discuss how exactly these 'proteomic' techniques might be relevant to a psychiatric disease, for example, schizophrenia. Schizophrenia, with its early developmental and later degenerative/atrophic components, is a particularly complex disorder. We know that multiple, largely unidentified genetic (Weinberger et al, 2001) and environmental (Cannon et al, 2003) risk factors interact to (Thompson et al, 2001). However, the underlying functional changes at the cellular level remain unknown. This is where the strength of a proteomics approach lies, allowing one to identify, quantify and compare the levels of thousands of proteins. The main methods encompassed within this term can be broadly described as those using two-dimensional gel electrophoresis and those using non-gel-based methods to separate and identify proteins in a complex mixture, such as a whole cell or tissue lysate.

\section{GEL-BASED PROTEOMIC METHODS}

Gel-based methods are the most commonly used and well-defined techniques in the proteomic world, having been around since the mid-1970s (O'Farrell, 1975). Briefly, two-dimensional gel electrophoresis involves the separation of solubilised proteins in the first dimension according to their lead to disease and disease progression

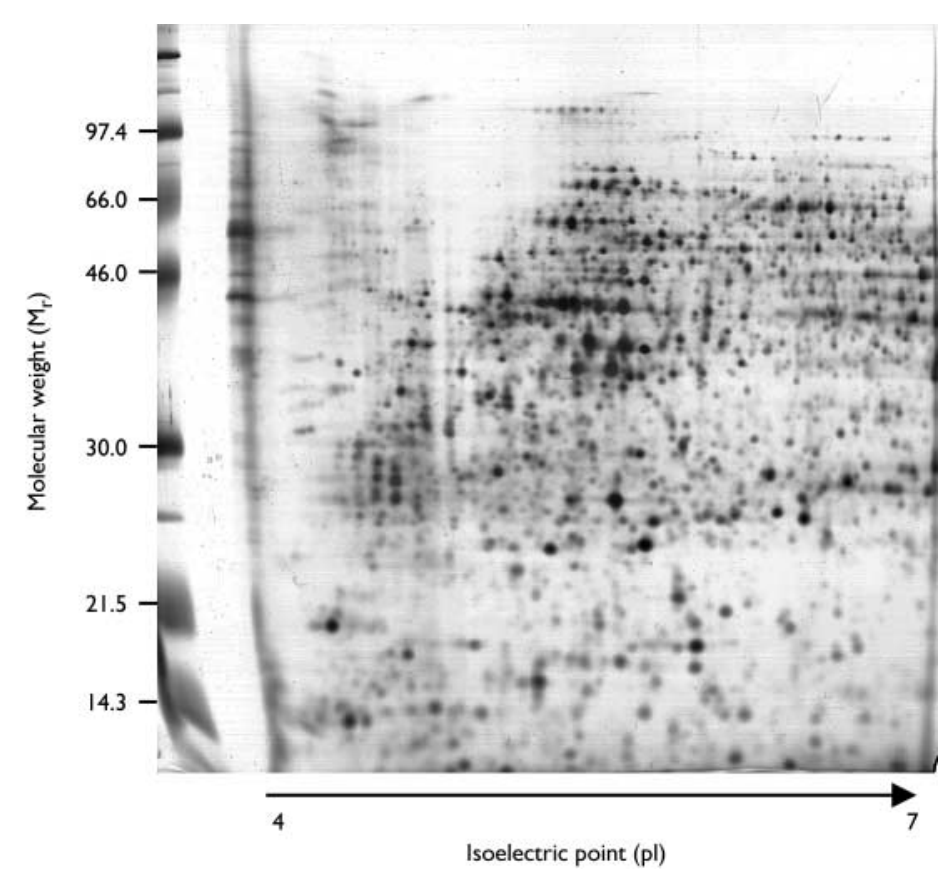

Fig. I Two-dimensional gel $(18 \times 20 \mathrm{~cm})$ of post-mortem brain tissue. Grey matter was removed from the dorsolateral prefrontal cortex $(\sim 100 \mathrm{mg})$ and homogenised. Extracted proteins were subsequently separated by isoelectric point in the first dimension and molecular weight in the second dimension. 
charge (isoelectric point, $\mathrm{p} I$ ), followed by their separation in the second dimension by sodium dodecyl sulphate-polyacrylamide gel electrophoresis (SDS-PAGE), according to their relative molecular mass $\left(\mathrm{M}_{\mathrm{r}}\right)$. The resulting protein 'maps' can then be compared using image analysis software and subsequent statistical analysis to identify protein spots of interest (Fig. 1). In addition, a mass spectrometry approach, for example matrix-assisted laser desorption/ionisation mass spectrometry, which measures peptide masses based on their mass-to-charge ratio, generates spectra that can be used to search for correlating protein identities in sequence databases. These techniques and the newer two-dimensional fluorescence difference gel electrophoresis method, which allows two samples labelled with different fluors to be resolved on the same gel, have been used in post-mortem studies of human brain tissue in schizophrenia, bipolar disorder and major depression (Edgar et al, 1999; Johnston-Wilson et al, 2000; Prabakaran et al, 2004).

A recent study investigated schizophrenia using post-mortem brain tissue at the transcriptome, metabolome and proteome level (Prabakaran et al, 2004). This approach highlights the complementary role of proteomics in validating and providing additional information on posttranslational processing to transcriptomic investigations. Two-dimensional fluorescence difference gel electrophoresis was used to compare protein expression in prefrontal cortical tissue taken from 10 controls and 10 individuals with schizophrenia. The combined findings strongly suggest a mitochondrial dysfunction within this area of the brain in schizophrenia. Proteomics was invaluable in leading to this conclusion, with almost $50 \%$ of the differentially expressed proteins being associated with mitochondrial function or oxidative stress. In support of this, our recent twodimensional gel electrophoresis study of the anterior cingulate cortex in schizophrenia, bipolar disorder and major depression also revealed abnormal expression of many proteins with roles in metabolism and mitochondrial function (Pennington $e t$ al, 2004).

\section{NON-GEL-BASED PROTEOMIC METHODS}

Gel-based methods have fundamental limitations due to their inability to represent the whole proteome. For example, although brain tissue is the most complex tissue in the body with regard to heterogeneity of protein content, the average two-dimensional gel can resolve only a few thousand proteins, and many of these represent different post-translationally modified forms of a much smaller number of gene products. Alternative approaches avoid the use of gels altogether by combining liquid chromatography and mass spectrometry. In these 'shotgun' proteomics approaches, the sample is separated by one or more dimensions of liquid chromatography to reduce the complexity of peptide fractions. These are subsequently introduced into a tandem mass spectrometer for sequence-based identification. Such techniques can hypothetically cope better with complex samples than the gelbased methods, have a higher throughput and generate data faster. However, these approaches cannot distinguish between different forms of the same protein due to processes such as post-translational modification. In addition, stable isotope labelling methods must be combined if differential quantitative analysis of two populations of proteins is needed. The most widely used isotope-coded affinity tag method can distinguish between two protein populations, for example disease $v$. control, where there is a 'light' reagent tag and a 'heavy' reagent tag to label total tryptic peptides generated from the two samples. The combined sample then undergoes single or multidimensional chromatography, and resultant peptide mass peaks from mass spectrometry clearly show the two samples separated by the mass of the two tags, allowing relative quantitation. This method has been used to study changes in protein expression in a cortical neuron cell culture undergoing DNA damage-induced death, where 150 proteins were identified and quantitated (Johnson et al, 2004). Fortyfive per cent of these had altered expression in the damaged neurons; validation was carried out for 14 of these, mostly cytoskeletal proteins and those involved in energy production and oxidative stress, using Western blots and immunohistochemistry.

In addition, there is much interest in the development of antibody and protein arrays for quantitative expression profiling, but considerable work remains to be carried out before this approach can be routinely used in proteomic investigations (Cutler, 2003). One chip-based method that has already had a major impact on the discovery of potential biomarkers, especially in cancer research, is surfaceenhanced laser desorption ionisation (SELDI) coupled with time-of-flight mass spectrometry (Petricoin et al, 2002). In this approach a small volume of a liquid sample, typically a body fluid such as serum, plasma or urine, is spotted onto a chemically modified surface of a special target. Using this approach it is possible to rapidly screen large numbers of samples to detect disease-associated proteins, but a major disadvantage is that it provides no direct information on the identities of these components. Nevertheless, this identification may not be required. For example, using bioinformatics, Petricoin et al (2002) used SELDI mass spectral patterns to discriminate normal serum samples from samples taken from patients with early ovarian cancer. Identification of disease occurred in nearly $100 \%$ of cases. However, re-analysis of these data has led to a questioning of the validity of this technique (Check, 2004) and has raised many methodological issues that need clarification before the technique can be applied to the investigation of neurological and psychiatric disorders.

\section{CREATING A SUB - PROTEOME}

It is of interest to fractionate samples at the cellular and sub-cellular level to increase proteomic coverage so that specific and less abundant subsets of the total proteome can be analysed. Laser-assisted micro-dissection allows the isolation of regions of interest that cannot be dissected by the naked eye, for example a sub-region of the hippocampus. This method has been successfully applied to the investigation of pancreatic cancer by Shekouh et al (2003) who removed tumour cells from surrounding tissue and performed two-dimensional gel electrophoresis and mass spectrometry identifying a novel tumour-associated protein, S100A6.

The sub-fractionation of homogenised brain samples allows isolation of membrane, mitochondrial or nuclear proteins. In addition, proteins can be isolated that have undergone specific post-translational modifications, such as phosphorylated proteins. When combined, such techniques result in decreased sample heterogeneity and thus increase the likelihood of gaining information about proteins of potential interest. 


\section{WHICH SAMPLES CAN WE USE?}

Most proteomic research into psychiatric disorders has focused on the comparative analysis of post-mortem brain tissue. This tissue has limitations in terms of differentiating primary and secondary pathology. Therefore, other tissues must also be investigated in order to obtain a fuller picture of disease-associated changes in protein profiles. Fortunately, proteomic techniques can be applied to whatever sample is of interest, for example animal models and drug action effects in vitro. Furthermore, with the advent of the fractionation techniques described previously, it will also be possible to study cerebrospinal fluid (CSF) or plasma and serum samples, fluids containing several high-abundance proteins of little interest, such as albumin. In addition to providing insights into the disease process, studying CSF using proteomic methods may allow researchers to gain further insights into drug action and effect. A two-dimensional gel study looking at the CSF of individuals with schizophrenia found an average $21 \%$ decrease in the number of proteins detected in those receiving haloperidol compared with CSF from the same individuals after withdrawal of treatment (Johnson et al, 1992).

The role of proteomics in psychiatric research is clear. It provides researchers with a set of tools to help validate and expand on both genetic and epigenetic studies in order to explore further the pathogenesis of disease. As this holistic approach is largely non-hypothesis based, it should reveal aspects of abnormal function previously inaccessible to researchers. In addition, from a clinical viewpoint, diagnostic biomarkers may be revealed through analysis of serum or CSF. These biomarkers will hopefully facilitate the identification of those at risk of psychosis, and treatment

K. PENNINGTON, BSc, Department of Psychiatry, Royal College of Surgeons, Beaumont Hospital, Dublin, and Proteome Research Centre, Conway Institute of Biomolecular and Biomedical Research, University College Dublin; D. COTTER, MRCPsych, PhD, Department of Psychiatry, Royal College of Surgeons, Beaumont Hospital, Dublin; M. J. DUNN, PhD, Proteome Research Centre, Conway Institute of Biomolecular and Biomedical Research, University College Dublin, Ireland

Correspondence: Ms K. Pennington, Department of Psychiatry, Royal College of Surgeons in Ireland, Beaumont, Dublin 9, Ireland. Tel: +353 I 8092750; fax: +353 I 8093098; e-mail: kpennington@rcsi. ie

(First received I2 July 2004, final revision I December 2004, accepted I December 2004)

response may also be predicted. We look forward to the clinical improvements that should follow the application of proteomic methods to psychiatric and neurological disorders.

\section{ACKNOWLEDGEMENTS}

We thank the Stanley Foundation Brain Consortium, The Wellcome Trust, the National Alliance for Research (in Schizophrenia and Affective Disorder), and Science Foundation Ireland for funding.

\section{REFERENCES}

Cannon, M., Kendell, R., Susser, E. S., et al (2003) Pre and perinatal risk factors for schizophrenia. In Epidemiology of Schizophrenia (eds R. M. Murray, P. B. Jones, E. S. Susser, et al), pp. 74-79. Cambridge: Cambridge University Press.

Check, E. (2004) Proteomics and cancer: running before we can walk? Nature, 429, 496-497.

Cutler, P. (2003) Protein arrays: the current state-of the-art. Proteomics, 3, 3-18.

Edgar, P. F., Schonberger, S. J., Dean, B., et al (1999) A comparative proteome analysis of hippocampal tissue from schizophrenic and Alzheimer's disease individuals. Molecular Psychiatry, 4, 173-178.

Johnson, G., Brane, D., van Kammen, D. P., et al (1992) Haloperidol induced CSF protein variations in schizophrenic patients: as studied by two-dimensional electrophoresis. Applied and Theoretical Electrophoresis, 3, $21-26$.

Johnson, M. D., Yu, L. R., Conrads, T. P., et al (2004) Proteome analysis of DNA damage-induced neuronal death using high throughput mass spectrometry. Journal of Biological Chemistry, 279, 26685-26697.
Johnston-Wilson, N. L., Sims, C. D., Hofmann, J-P., et al (2000) Disease-specific alterations in frontal cortex brain proteins in schizophrenia, bipolar disorder, and major depressive disorder. Molecular Psychiatry, 5 142-149.

O'Farrell, P. H. (1975) High resolution two dimensional electrophoresis of proteins. Journal of Biological Chemistry, 250, 4007-4021.

Pennington, K., Beasley, C. B., Dunn, M., et al (2004) Proteome analysis of the anterior cingulate cortex in major psychiatric disorders. Biological Psychiatry, 55, 132-133.

Petricoin, E. F., Ardekani, A. M., Hitt, B. A., et al (2002) Use of proteomic patterns in serum to identify ovarian cancer. Lancet, 359, 572-577.

Prabakaran, S., Swatton, J. E., Ryan, M. M., et a (2004) Mitochondrial dysfunction in schizophrenia: evidence for compromised brain metabolism and oxidative stress. Molecular Psychiatry, 9, 684-697.

Shekouh, A. R., Thompson, C. C., Prime, W., et al (2003) Application of laser capture microdissection combined with two-dimensional electrophoresis for the discovery of differentially regulated proteins in pancreatic ductal adenocarcinoma. Proteomics, 3, 1988-2001.

Thompson, P. M., Vidal, C., Giedd, J. N., et al (200I) Mapping adolescent brain change reveals dynamic wave of accelerated gray matter loss in very early-onset schizophrenia. Proceedings of the National Academy of Sciences of the USA, 98, I1650-11655.

Wasinger,V. C., Cordwell, S. J., Cerpa-Poljak, A., et al (1995) Progress with gene-product mapping of the Mollicutes: Mycoplasma genitalium. Electrophoresis, 16, 1090-1094.

Weinberger, D. R., Egan, M. F., Bertolino, A., et al (200I) Prefrontal neurons and the genetics of schizophrenia. Biological Psychiatry, 50, 825-844. 